\title{
Improved outcome of acute myeloid leukaemia in Down's syndrome
}

\author{
J L Craze, G Harrison, K Wheatley, I M Hann, J M Chessells
}

\begin{abstract}
Objective-To review the clinical features, treatment, and outcome of children in the UK with Down's syndrome and acute myeloid leukaemia (AML).

Design-A retrospective study of 59 children with Down's syndrome and AML presenting between 1987 and 1995. Data were obtained from hospital case notes, trial records, and by questionnaire.

Results-The patients were unusually young (median age, 23 months) with a predominance of megakaryoblastic AML. Two of the seven infants who presented with abnormal myelopoesis aged 2 months or younger achieved complete spontaneous remission. Most of the older children with AML (32 of 52) were treated on recognised intensive protocols but 13 received individualised treatment and seven symptomatic treatment alone. Only four received a bone marrow transplant (BMT) in first remission. For the 45 older children who received chemotherapy the overall survival was $55 \%$ (median follow up 4.5 years). Patients on individualised protocols had a similar overall survival and toxic death rate but marginally higher relapse rate than those on standard (intensive) protocols. Children with Down's syndrome treated on the national AML 10 trial had a similar overall survival $(70 \% v$ $59 \%$ ) at five years to children of comparable age without Down's syndrome: their improved relapse risk $(12 \% v 38 \%)$ offset the slight increase in deaths as a result of treatment toxicity $(19 \% v 11 \%)$.

Conclusion-Neonates with Down's syndrome and abnormal myelopoesis may achieve spontaneous remission, and older children with Down's syndrome and AML can be treated successfully with intensive chemotherapy, without BMT.

(Arch Dis Child 1999;81:32-37)
\end{abstract}

Keywords: Down's syndrome; acute myeloid leukaemia; treatment; outcome

Children with Down's syndrome have an increased incidence of both acute lymphoblastic and acute myeloid leukaemia (AML). ${ }^{12}$ Neonates with Down's syndrome may also develop features of leukaemia that undergo spontaneous remission, so called transient abnormal myelopoesis (TAM). ${ }^{3}$ In the past, children with Down's syndrome and AML may have received supportive care only, ${ }^{4-7}$ or died during treatment from infection, ${ }^{5}$ but recent studies suggest that they can tolerate and respond well to intensive treatment. ${ }^{489} \mathrm{We}$ reviewed the clinical features, treatment trends, and outcome for children with Down's syndrome and AML in the UK since the advent of more intensive treatment schedules.

\section{Patients and methods}

We obtained information about all children with Down's syndrome developing AML during the years 1987-95 when the national Medical Research Council (MRC) trial for childhood AML, MRC AML 10 was accruing patients. ${ }^{10}$ Cases were ascertained from the population based registry of children with cancer at the Childhood Cancer Research Group (CCRG) Oxford, with permission from the United Kingdom Children's Cancer Study Group (UKCCSG) epidemiology and registry group. Sixty three cases were identified from the register: four were not cared for by UKCCSG members and therefore were excluded, leaving a study group of 59. Information about these children was collected from the hospital case notes of those treated at Great Ormond Street Children's Hospital, London (GOSH); from trial records held at the Clinical Trial Service Unit, Oxford of those treated on the MRC AML 10 trial, and by questionnaire from the consultants responsible for the remaining children.

We recorded data on the child's general health, any preceding haematological abnormalities, and clinical and laboratory features at presentation with leukaemia. Details of all treatment received, any serious complications, and the outcome in terms of achievement of remission, occurrence of relapse, or death and cause of death were ascertained.

Figure 1 shows the distribution of the patients by age and treatment received. Children aged 2 months or less were excluded from the survival analyses because of the difficulties of differentiation from TAM in this group. To assess the outcome after different types of treatment, older children who received chemotherapy were divided into three groups (fig 1): group A $(n=24)$, the AML 10 patients; group $\mathrm{B}(\mathrm{n}=8)$, those who had received any other recognised (intensive) protocol; and group C $(n=13)$, those treated with any individualised protocol. The 11 children with Down's syndrome treated at GOSH received the four standard courses of chemotherapy from the MRC AML 10 protocol without randomisation or high dose treatment: this conformed to one possible arm of the MRC AML 10 trial. Therefore, these children were grouped with the 13 children with Down's syndrome who had been registered on the MRC AML 10 trial 


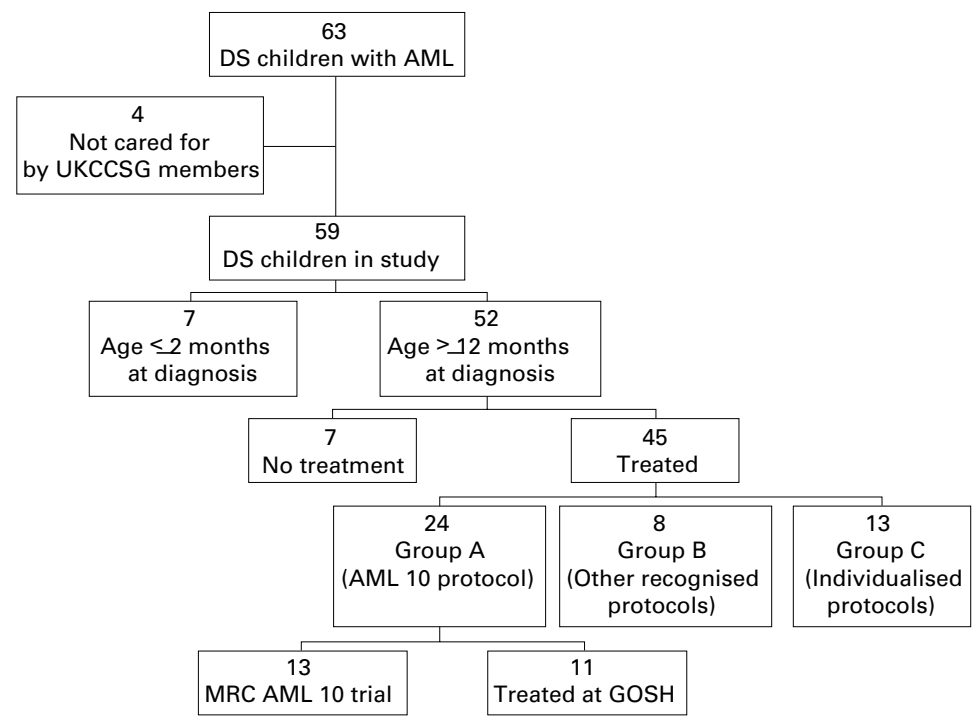

Figure 1 Division of children with Down's syndrome (DS) and acute myeloid leukaemia $(A M L)$. GOSH, Great Ormond Street Hospital; UKCCSG, United Kingdom Children's Cancer Study Group.
Down's syndrome. Fisher's exact test was used to test for differences in remission rate, incidence of previous haematological problems, or morphological subtype; the MantelHaenszel test for trend was used to test for differences in presenting white cell count between children with Down's syndrome and controls. Kaplan-Meier life tables were constructed for survival data and were compared by means of the log rank test, ${ }^{12}$ surviving patients being censored at 1 September 1996, when follow up was complete for all subjects. Survival estimates are given in the text as percentages with standard error in parentheses. All $\mathrm{p}$ values are two tailed.

\section{Results}

CLINICAL FEATURES

Among the 59 patients there were 29 boys and 30 girls; their median age at presentation was 23 months (0-174 months). There were two distinct age clusters: seven infants aged 2 months or less and all but two of the others presenting between 12 and 42 months (two older children were 61 and 174 months). Thirty one children had additional medical problems including 24 with congenital heart disease (seven corrected, 17 uncorrected) and five with previous severe upper airway problems or recurrent pneumonia.

Table 1 shows the haematological features at presentation for all patients with Down's syndrome and for controls of similar age on MRC AML 10. In general, children with Down's syndrome had low leucocyte counts (median, $\left.7.6 \times 10^{9} / 1\right)$. Morphological classification according to Franco-American-British (FAB) criteria $^{13}{ }^{14}$ showed a predominance of megakaryoblastic leukaemia (M7). Comparison of children with Down's syndrome in group A (excluding the child aged 5 years) with controls of similar age on MRC AML 10 revealed that the children with Down's syndrome had a lower presenting white cell count ( $p<0.001$ ), a higher incidence of the M7 subtype $(p<0.001)$, and a higher incidence of preceding haematological problems than did controls $(p<0.001)$.

Seventeen of the children aged 12 months or more at diagnosis had a prodromal period of thrombocytopenia, with or without anaemia, for a median of 5 months (1.5 to 17 months) before developing frank AML. Five of these years, compared with $67 \%$ of those without

Table 1 Patient characteristics

\begin{tabular}{|c|c|c|c|c|c|c|c|}
\hline & \multicolumn{3}{|c|}{ Presenting white blood count $\left(\times 10^{9} / l\right)$} & \multicolumn{2}{|c|}{ FAB type } & \multirow[b]{2}{*}{ TAMt } & \multirow[b]{2}{*}{ Prodrome $\neq$} \\
\hline & $<10$ & $10-50$ & $>50$ & M7 & Other & & \\
\hline \multicolumn{8}{|c|}{ Down's syndrome patients by age at presentation } \\
\hline 2 months or younger $(n=7)$ & 0 & $4(57)$ & $3(43)$ & $7(100)$ & 0 & & \\
\hline 12 months or older $(n=52)$ & $37(71)$ & $10(19)$ & $4(8)$ & $29(56)$ & $23(44)$ & $5(10)$ & $17(33)$ \\
\hline \multicolumn{8}{|l|}{ AML10 patients younger than 4 years } \\
\hline Down's syndrome (group $\mathrm{A}, \mathrm{n}=23$ ) $\sqrt{ }$ & $15(65)$ & $6(26)$ & $2(9)^{\star \star \star}$ & $15(65)$ & $8(35)^{\star \star \star}$ & $3(13)$ & $10(43)^{\star \star \star}$ \\
\hline Non-Down's syndrome $(\mathrm{n}=114)$ & $30(26)$ & $53(46)$ & $30(26)$ & $15(13)$ & $98(86)$ & & $3(3)$ \\
\hline
\end{tabular}

Values are $\mathrm{n}(\%)$

†Previous, resolved, abnormal blood count in the neonatal period.

$\ddagger$ Abnormal blood count for at least four weeks before developing true AML.

OOne child with Down's syndrome in the AML 10 trial is older than 4 years and is excluded from this particular table and analysis. $\star \star \star \mathrm{p}<0.001$; $\mathrm{p}$ values are for the Mantel-Haenszel test (trend in presenting white blood count) and Fisher's exact test (incidence of FAB type), Down's syndrome children on AML 10 compared with non-Down's syndrome controls.

AML, acute myeloid leukaemia; FAB, Franco-American-British criteria; TAM, transient abnormal myelopoesis. 
Table 2 Cytogenetic changes in bone marrow among 48 children with Down's syndrome for whom cytogenetic results are available

\begin{tabular}{lc}
\hline Anomaly & Frequency \\
\hline Constitutional trisomy 21 only* & 15 \\
Tetrasomy 21 or pentasomy 21 & 4 \\
Trisomy 8 & 15 \\
Other simple trisomies $†$ & 10 \\
Chromosome loss & 5 \\
Balanced translocation & 6 \\
Unbalanced translocation $\ddagger$ & 9 \\
Monosomy 7 & 2 \\
Partial deletion $\$$ & 11 \\
Other structural changes & 6 \\
\hline
\end{tabular}

$\star$ Regular trisomy $21(\mathrm{n}=14)$; $\mathrm{t}(21 \mathrm{q} ; 21 \mathrm{q})(\mathrm{n}=1)$.

†Chromosomes 11 in four cases.

$\ddagger$ Six involving chromosome 1 .

§Two involving chromosome 7 ; five involving chromosome 6 ; four others.

had previously been diagnosed as having TAM in the neonatal period (table 1).

Bone marrow cytogenetics were available for 48 patients. All showed constitutional trisomy $21 ; 15$ showed no other anomaly. The most common additional anomaly was trisomy 8 and many of the abnormalities were complex (table 2 ). None of the karyotypes showed any of the changes associated with a favourable outcome in AML: $t(15 ; 17)$, inv(16), or $t(8 ; 21)$. Two showed monosomy 7 , which is associated with an unfavourable prognosis.

INFANTS

Characteristics of the seven infants aged 2 months or less are given in tables 1 and 3. Both those who presented at birth were premature. Only one, who presented aged 1 month with a hemiparesis, received chemotherapy (modified AML 10 protocol): he died from infection. Three were very sick and died from their haematological disease after only 0-9 days, receiving supportive care only. However, two babies underwent complete spontaneous remission and remain well, and one was stable haematologically without treatment but died from other medical problems.

TREATMENT RECEIVED, CHILDREN AGED 12 MONTHS OR MORE

Seven children aged 12 months or more received supportive care only; all died (median survival, 55 days; range 9-274). Their median age was 22 months; only two had other medical problems (one having severe cardiac disease).

The remaining 45 older children received chemotherapy (fig 1). Several modifications were made to the MRC AML 10 protocol for the children in group A: in particular, two patients received only three courses of chemo-

Table 3 Outcome for seven children with Down's syndrome aged 2 months or less at presentation

\begin{tabular}{lllll}
\hline Patient & $\begin{array}{l}\text { Age at presentation } \\
\text { (days) }\end{array}$ & Treatment & $\begin{array}{l}\text { Remission } \\
\text { achieved }\end{array}$ & Outcome \\
\hline 1 & 33 & $\begin{array}{l}\text { Intensive } \\
\text { chemotherapy }\end{array}$ & Yes & $\begin{array}{l}\text { Died, pneumonia and pericardial } \\
\text { effusion }\end{array}$ \\
2 & 6 & None & No & Died, progressive disease \\
3 & 0 & None & No & Died, progressive disease \\
4 & 10 & None & No & Died, Hirschprung's enterocolitis \\
5 & 0 & None & No & Died, progressive disease \\
6 & 71 & None & Yes & Well at 1 year \\
7 & 28 & None & Yes & Well at 7 years \\
\hline
\end{tabular}

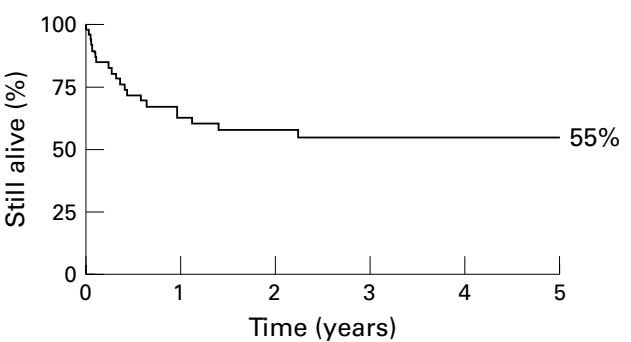

Figure 2 Overall survival of 45 children with Down's syndrome aged 1 year or over receiving chemotherapy.

therapy because of excessive toxicity (both remain in remission). Only minor modifications were made to the standard protocols used for children in group B. The intensity of treatment designed for the children in group $\mathrm{C}$ varied considerably, some being outpatient based. Only four children who received chemotherapy had a BMT from a histocompatible sibling donor: three in group $\mathrm{A}$ and one in group $\mathrm{B}$.

\section{OUTCOME OF TREATMENT}

Thirty eight of the 45 older children who received chemotherapy achieved complete remission: six died during the first course of treatment and only one had resistant disease. Six children subsequently died in remission and nine relapsed: two of these received further chemotherapy, one achieving durable second remission. At a median follow up of 4.5 years (range 1.0-9.7), the overall five year survival was $55 \%$ (SE, $7.5 \%$ ) for the 45 children aged more than 1 year receiving any chemotherapy (fig 2).

Tables 4 and 5 compare the results for those who received any protocol (intensive) treatment (groups A and B) with those who received individual treatment (group C). There is no apparent difference between groups A and $B$ combined and group $C$ in remission rate $(\mathrm{p}=0.7)$ or overall survival $(\mathrm{p}=0.9)$, but the lack of randomisation, small numbers, and the heterogeneity of treatments render formal analysis of difference in outcome unreliable. However, the risk of relapse and causes of death differ between the two groups. Those on protocol treatments have a lower risk of relapse at five years $(p=0.08)$ than those on individual treatment. Most deaths in groups A and B were the result of treatment toxicity, whereas most deaths in group $\mathrm{C}$ resulted from relapsed or resistant disease (table 5).

Analysis of outcome for children with and without Down's syndrome on MRC AML 10 showed no difference in remission rate between children with Down's syndrome (group A, excluding the child aged over 4 years) and controls of similar age $(91 \% v 95 \% ; \mathrm{p}=0.6)$. The overall five year survival for children with Down's syndrome (70\%; SE, 9.6\%) was greater than for controls (59\%; SE, 4.6\%) (fig $3)$, although this was not significant $(\mathrm{p}=0.6)$. The children with Down's syndrome had a lower relapse risk than controls $(12 \%$ (SE, $7.8 \%) v 38 \%(5.1 \%) ; \mathrm{p}=0.04)($ fig 4$)$. 
Table 4 Outcome for 45 children with Down's syndrome aged 1 year or older receiving chemotherapy

\begin{tabular}{lllll}
\hline Type of treatment & $n$ & $\begin{array}{l}\text { Number } \\
\text { remitting (\%) }\end{array}$ & $\begin{array}{l}\text { Overall survival at } \\
5 \text { years, \% (SE) }\end{array}$ & $\begin{array}{l}\text { Relapse risk at 5 } \\
\text { years, \% (SE) }\end{array}$ \\
\hline Any & 45 & $38(84)$ & $55(7.5 \%)$ & $26(8.0 \%)$ \\
On protocol (groups A and B) & 32 & $26(81) \|^{\star}$ & $59(8.7 \%) \dagger^{\star \star}$ & $16(8.3 \%)^{\star \star \star}$ \\
Individual (group C) & 13 & $12(92) \S$ & $43(14.7 \%) \ddagger$ & $46(15.0 \%)$ \\
\hline
\end{tabular}

${ }^{\star} \mathrm{p}=0.7 ;{ }^{\star \star} \mathrm{p}=0.9 ;{ }^{\star \star \star} \mathrm{p}=0.08 ; \mathrm{p}$ values are for Fisher's exact test (remission rate and deaths in remission) and log rank test (overall survival and relapse risk).

tOne alive in relapse; †one alive in second complete remission; \single remission failure as a result of resistant disease; $₫$ all remission failures as a result of death during first course of treatment.

Table 5 Causes of death among 45 children with Down's syndrome aged 1 year or older receiving chemotherapy

\begin{tabular}{lllll}
\hline & \multicolumn{4}{c}{ Deaths } \\
\cline { 3 - 5 } Type of treatment & Patients & Total & $\begin{array}{l}\text { Caused by } \\
\text { disease* }\end{array}$ & $\begin{array}{l}\text { Caused by treatment } \\
\text { toxicityt }\end{array}$ \\
\hline On protocol (groups A and B) & 32 & 13 & 2 & 11 \\
Individual (group C) & 13 & 7 & 6 & $1 \neq$
\end{tabular}

*Deaths caused by resistant or relapsed disease; tdeaths caused by sepsis or cardiomyopathy; ¥single death caused by cardiomyopathy.

MORBIDITY OF TREATMENT

Thirty four of the 45 children receiving chemotherapy had at least one serious complication of treatment, predominantly infection of the respiratory tract, severe upper airways obstruction, or gastrointestinal toxicity. Three required admission to an intensive treatment unit and three had their treatment modified because of severe toxicity. The four patients who received an allograft all survived without apparent excessive morbidity. In general, the rate of remission deaths reflects treatment toxicity; this was greater for children with Down's syndrome than for controls of similar age treated on AML 10 (19\% v 11\%), but this difference was not significant $(\mathrm{p}=0.3)$.

There were four episodes (one fatal) of cardiac failure thought to be caused by anthracycline induced cardiomyopathy. Follow up echocardiographic data are incomplete, but of the nine children with Down's syndrome and

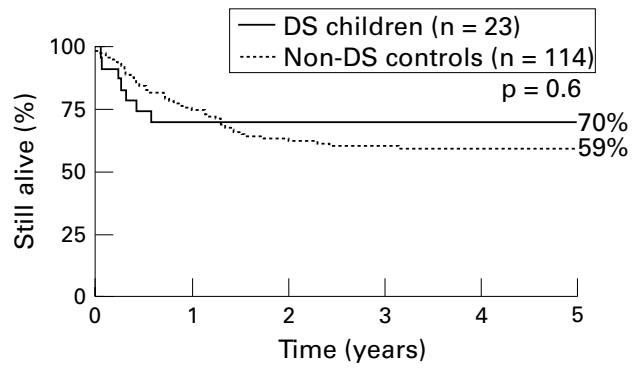

Figure 3 Overall survival of children in the AML 10 trial aged under 4 years by Down's syndrome status.

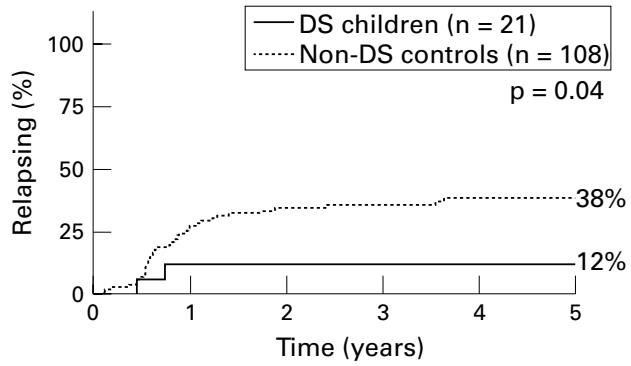

Figure 4 Relapse risk of children in the AML 10 trial aged under 4 years by Down's syndrome status. cardiomyopathy or asymptomatic deterioration in function on echocardiography, four (44\%) had congenital heart disease. This is comparable with the $42 \%$ prevalence of heart disease among all children with Down's syndrome who received chemotherapy.

\section{Discussion}

This registry based series includes $94 \%$ of children with Down's syndrome presenting with AML in the UK over a nine year period. We have confirmed a characteristic clinical picture-young age, low white cell count, a predominance of $\mathrm{M} 7 \mathrm{AML}$, and a prodromal myelodysplastic period-features that are relatively uncommon in childhood AML but are well described in patients with Down's syndrome. $^{7-9} 1516$ The M7 (megakaryoblastic) variant of AML, seen in only $5-10 \%$ of childhood AML, ${ }^{6}{ }^{17}$ is the most frequent subtype in children with Down's syndrome. ${ }^{891618}$

The phenomenon of TAM in the neonate with Down's syndrome is well known. ${ }^{318}$ Our study was not designed to ascertain all cases of TAM and the true frequency is unknown because milder cases may remain undetected or not notified to the childhood cancer registry. In a retrospective survey of this type, we cannot be certain of the differentiation between TAM and true AML, but two of our patients had spontaneously resolving disease and would conform to a diagnosis of TAM. Three, who were all were very sick at diagnosis, had a fatal outcome as has been described by others. ${ }^{3}$ All neonates with Down's syndrome and apparent leukaemia who are well should initially be monitored in the expectation that many recover spontaneously. Only $20-30 \%$ of those with TAM subsequently develop AML, ${ }^{3}$ often after a period of myelodysplasia. Although in TAM the abnormal clone seems to "burn out", ${ }^{19}$ AML is fatal if untreated.

Our study shows that children with Down's syndrome can tolerate intensive chemotherapy and, despite treatment toxicity, have a favourable relapse risk and a similar overall survival to other children. This represents an improvement over the last decade, ${ }^{7}$ and confirms results of other studies, some of which have shown a better outcome for children with Down's syndrome than for other children when treated with standard chemotherapy. ${ }^{48916}$

Despite this, and despite changing attitudes to children with Down's syndrome, ${ }^{416}$ our study also confirms that there is still some reluctance to offer standard chemotherapy to children with Down's syndrome. ${ }^{45}$ Individual treatment is sometimes offered, presumably with the aim of minimising toxicity, but the numbers involved are too small to demonstrate any reduction in toxicity and the relapse risk increases. Therefore, overall survival is not obviously improved. Although intensive treatment causes significant toxicity, to which children with Down's syndrome may be especially vulnerable, because of relative immunodeficiency ${ }^{20}$ and pre-existing morbidity, we have confirmed that they can tolerate such chemotherapy with a favourable outcome. 
Therefore, we suggest that children with Down's syndrome should receive standard chemotherapy, without BMT. Good supportive care is vital.

Concern about the potential cardiotoxicity of anthracyclines may contribute to reluctance to offer standard treatment to children with Down's syndrome. ${ }^{6}$ There is no evidence that the presence of congenital heart disease increases the risk of anthracycline toxicity, ${ }^{8}$ and although our data on cardiac outcome are incomplete, we found no evidence for an increased risk among those with congenital heart disease. We suggest anthracyclines can be used for children with Down's syndrome although, as for all children, reduced anthracycline exposure would be ideal and may become feasible using one of the newer regimens exploring the use of other drug combinations.

Why do children with Down's syndrome have such a low relapse risk? The relatively young age of the children is unlikely to be important because age is not related to prognosis in childhood AML, ${ }^{21}$ and we used controls of a similar age for the AML 10 analysis. The lower white cell count of children with Down's syndrome at presentation may be relevant because some studies have shown a trend to poorer outcome with higher leucocyte counts in AML. ${ }^{61}$ However, in the MRC AML 10 trial as a whole, the presenting count had no influence on overall survival. There is no evidence that M7 is a "favourable" subtype of AML. ${ }^{4} 61622$ The best prognostic indicator in childhood AML is the karyotype of the leukaemic blast cells. ${ }^{23}$ One of the favourable abnormalities $\mathrm{t}(15 ; 17)$, $\operatorname{inv}(16)$, or $\mathrm{t}(8 ; 21)$ is found in $\sim 30 \%$ of cases of childhood AML. ${ }^{24}$ None were seen in our study, in keeping with other series of children with Down's syndrome and AML. ${ }^{516}$ Two children in our study (4\%) had the unfavourable karyotype monosomy 7 , similar to the $5 \%$ prevalence in other series of patients with AML. ${ }^{24}$ Therefore, the characteristics of AML seen in the child with Down's syndrome do not necessarily predict a low relapse risk. We have to conclude that constitutional trisomy 21 increases the risk of developing AML but simultaneously acts as a favourable prognostic factor.

The lower relapse risk might relate to changes in purine nucleotide synthesis and folate metabolism within cells with trisomy 21 . Theoretically, this should increase the sensitivity of the cells to the therapeutic effects of cytarabine, one of the drugs most active against AML. ${ }^{25}{ }^{26}$ Some of these changes have been demonstrated in vitro in megakaryoblasts from people with Down's syndrome, ${ }^{27}$ and remission has been achieved with very low dose cytarabine in children with Down's syndrome. ${ }^{15} 28$ However, it is likely that other unidentified factors also contribute to the low relapse risk.

We have confirmed the unique clinical and cytogenetic aspects of AML in children with Down's syndrome. Neonates may not require any treatment and we suggest that, if well, they should initially be monitored without treatment. With intensive treatment and adequate support, older children have a low relapse risk and BMT does not appear to be necessary for cure. There seems little justification in using individualised protocols. Our practice is to offer these children treatment with a modification of the current MRC AML 12 trial protocol without randomisation, avoiding high anthracycline doses. Provision of adequate supportive care is vital and improvements in such care may lead to an even better outcome in the future.

The authors acknowledge the assistance of G Draper, K Bunch, and C Stiller of the Childhood Cancer Research Group, Oxford and C Harrison of the cancer cytogenetics laboratory, Christie Hospital, Manchester. JLC and JMC were supported by the Hospital, Manchester. JLC
Leukaemia Research Fund.

1 Krivit W, Good RA. Simultaneous occurrence of mongolism and leukaemia. Am 7 Dis Child 1957;94:289-93.

2 Miller RW. Persons with exceptionally high risk of leukaemia. Cancer Res 1967;27:2420-3.

3 Zipursky A, Poon A, Doyle J. Leukemia in Down syndrome: a review. Pediatr Hematol Oncol 1992;9:139-49.

4 Lie SO, Jonmundsson G, Mellander L, Siimes MA, Yssing M, Gustaffson G, on behalf of the Nordic Society of Paediatric Haematology and Oncology. A population-based study of 272 children with acute myeloid leukaemia treated on two consecutive protocols with different intensity: best outcome in girls, infants, and children with Down's syndrome. Br Э Haematol 1996;94:82-8.

5 Creutzig U, Ritter J, Vormoor J, et al. Myelodysplasia and acute myelogenous leukemia in Down's syndrome. A report of 40 children of the AML-BFM study group. Leukemia 1996;10:1677-86.

6 Stiller CA, Eatock EM. Survival from acute nonlymphocytic leukaemia, 1971-88: a population based study. Arch Dis Child 1994;70:219-23.

7 Levitt GA, Stiller CA, Chessells JM. Prognosis of Down's syndrome with acute leukaemia. Arch Dis Child 1990;65: syndrome

8 Ravindranath Y, Abella E, Krischer JP, et al. Acute myeloid leukaemia in Down's syndrome is highly responsive to chemotherapy: experience on pediatric oncology group AML study 8498. Blood 1992;80:2210-14.

9 Kojima S, Kato K, Matsuyama T, Yoshikawa T, Horibe K. Favorable treatment outcome in children with acute myeloid leukemia and Down syndrome. Blood 1993;81: 3164.

10 Hann IM, Stevens RF, Goldstone AH, et al, on behalf of the Adult and Childhood Leukaemia Working Parties of the Medical Research Council. Randomized comparison of DAT versus ADE as induction chemotherapy in children and younger adults with acute myeloid leukaemia. Results AML 10). Blood 1997;89:2311-18.

11 Creutzig U, Ritter J, Zimmermann M, Schellong G, for the AML-BFM Study Group. Does cranial irradiation reduce the risk for bone marrow relapse in acute myelogenous the risk for bone marrow relapse in acute myelogenous ogenous leukemia study BFM-87. F Clin Oncol 1993;11: 279-86.

12 Peto R, Pike MC, Armitage P, et al. Design and analysis of randomized clinical trials requiring prolonged observation of each patient. Br F Cancer 1977;35:1-39.

13 Bennett JM, Catovsky D, Daniel MT, et al. Proposed revised criteria for the classification of acute myeloid leukaemia. Ann Intern Med 1985;103:620-5.

14 Bennett JM, Catovsky D, Daniel MT, et al. Criteria for the diagnosis of acute leukaemia of megakaryocytic lineage (M7). Ann Intern Med 1985;103:460-2.

15 Zipursky A, Thorner P, De Harven E, Christensen H, Doyle . Myelodysplasia and acute megakaryoblastic leukemia in Down's syndrome. Leuk Res 1994;18:163-71.

16 Lange BJ, Kobrinsky N, Barnard DR, et al. Distinctive demography, biology and outcome of acute myeloid leukemia and myelodysplastic syndrome in children with Down mia and myelodysplastic syndrome in children with Down Blood 1998;91:608-15.

17 Lie SO. Treatment of acute myeloid leukaemia in children. n: Chessells JM, Hann IM, eds. Bailliere's clinical paediatrics: leukaemia and lymphoma. London: Bailliere Tindall, 1995:757-78.

18 Hayashi Y, Eguchi M, Sugita K, et al. Cytogenetic findings and clinical features in acute leukemia and transient myeloproliferative disorder in Down's syndrome. Blood 1988;72:15-23.

19 Bain BJ. Transient leukaemia in newborn infants with Down's syndrome [letter]. Leuk Res 1994;18:723-4.

20 Report of a WHO scientific group. Primary immunodeficiency diseases. Clin Exp Immunol 1995;99(suppl 1):1-21.

21 Hurwitz CA, Schell MJ, Pui C-H, Crist WM, Behm F, Mirro J. Adverse prognostic features in 251 children treated for acute myeloid leukemia. Med Pediatr Oncol 1993;21:1-7.

22 Ribeiro RC, Oliveira MSP, Fairclough D, et al. Acute megakaryoblastic leukaemia in children and adolescents: a retrospective analysis of 24 cases. Leuk Lymphoma 1993;10: rospective

23 Swansbury GJ, Lawler SD, Alimena G, et al. Long-term survival in acute myelogeneous leukemia: a second 
follow-up of the fourth international workshop on chromo24 Raimondi SC, Kalwinsky DK, Hayashi Y, Behm FG, Mirro Raimondi SC, Kalwinsky DK, Hayashi Y, Behm FG, Mirro
J, Williams DL. Cytogenetics of childhood acute nonlymJ, Williams DL. Cytogenetics of childhood acute nonlym-
phocytic leukemia. Cancer Genet Cytogenet 1989;40:13-27. phocytic leukemia. Cancer Genet Cytogenet 1989;40:13-27.
25 Peeters M, Poon A. Down syndrome and leukaemia: unusual clinical aspects and unexpected methotrexate sensitivity. Eur F Pediatr 1987;146:416-22.

26 Garré ML, Relling MV, Kalwinsky D, et al. Pharmacokinetics and toxicity of methotrexate in children with Down syndrome and acute lymphocytic leukemia. 7 Pediatr 1987 111:606-12.
27 Taub JW, Matherly LH, Stout ML, Buck SA, Gurney JG, Ravindranath Y. Enhanced metabolism of 1- $\beta-D-$ arabinofuranosylcytosine in Down syndrome cells: a contributing factor to the superior event free survival of Down syndrome children with acute myeloid leukaemia. Blood 1996;87:3395-403.

28 Tchernia G, Lejeune F, Boccara J-F, Denavit M-F, Dommergues J-P, Bernaudin F. Erythroblastic and/or megakaryoblastic leukemia in Down syndrome: treatment with low-dose arabinosyl cytosine. If Pediatr Hematol Oncol 1996;18:59-62.

\section{Inflicted head injury-“could do better"}

It is almost 40 years since Dr Henry Kempe and his colleagues in Denver shocked the world with their paper "The battered child syndrome" (Fournal of the American Medical Association 1962;181:1724) but still paediatricians there are able to comment on the failure of doctors to make the diagnosis of child abuse. They report (Fournal of the American Medical Association 1999;281:621-6) that of 173 children under 3 years seen at the Denver Children's Hospital during 1990-95 with inflicted head injury, $54(31 \%)$ had been seen by doctors who failed to make the diagnosis. In these cases the diagnosis of abuse was delayed by up to 6 months (mean 7 days, range $<1-189$ days). The children in whom the diagnosis was not made seem to reflect doctors' preconceptions; they were more often young infants, white, living with both parents, and without breathing difficulties or seizures. Missing or misinterpreting $x$ ray abnormalities contributed to the difficulty in seven cases.

Of the 54 children whose diagnosis was missed $15(28 \%)$ were injured again, $22(41 \%)$ suffered complications related to the missed diagnosis, five died, and four of the deaths were thought to have been preventable. Nevertheless, the mortality in recognised cases $(17 \%)$ was greater than in missed cases $(9 \%)$, clearly because milder cases are more likely to be missed. Even in Denver, where doctors must have been exposed to as much publicity about child abuse as anywhere in the world, the diagnosis is still missed and a $\mathcal{F} A M A$ editorial writer can still comment "physicians can do better" (Ibid: 657-9)

ARCHIVIST 